\title{
Development of an Adaptive Collaborative Serious Game Based on Learning Style, Using Trace and Agent Technology
}

\author{
Hadya Boufera ${ }^{1}$, Fatima Bendella ${ }^{2}$, Karim Sehaba ${ }^{3}$ \\ 1,2 Université de science et de la Technologie d'Oran Mohamed Boudiaf \\ USTO-MB, BP 1505, El M'naour, 31000 Oran Algérie \\ hadya.boufera@univ-usto.dz \\ Fatima.bendella@univ-usto.dz \\ ${ }^{3}$ Université Lyon, LIRIS UMR5205 F-69676, France \\ karim.sehaba@liris.cnrs.fr
}

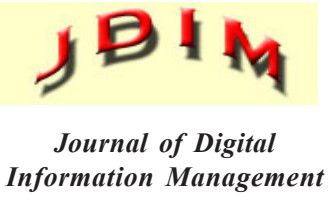

\begin{abstract}
In the collaborative serious game, adaptation is one of the main factors could be considered in the phase of conception since they can contribute to improve learning performance. Learning styles present one of the most factors that affect learning outcomes. Game-based learning (GBL) that takes into account learning styles in the process of adaptation, it furnishes to learners a greater benefit owing in the personalization of learning and contents materials according to their proper learning styles. In this paper, Adaptive system that personalize a collaborative serious game based on learning style proposed by Felder and Silverman is described. This system used trace and agent technology. A model of trace and group model are developed to extract adaptation knowledge in order to form plan that will be executed by Npc player. To evaluate a system, an experiment has been conducted on group of students. From the experimental results, it is concluded that the adaptive collaborative serious game based on learning style improve learning outcomes and effectiveness, and promote learning motivation.
\end{abstract}

Subject Categories and Descriptors

K.3.1 Computer Uses in Education] Collaborative learning I.2.6 [Learning] Knowledge acquisition

General Terms: Collaborative Learning, Game Based Learning, Learning Style

Keywords: Collaborative Learning, Serious Game, Adaptation, Learning Style, Trace, Npc Player

Received: 2 December 2017, Revised 18 December 2017, Ac- cepted 6 January 2018

DOI: $10.6025 / \mathrm{jdim} / 2018 / 16 / 3 / 127-135$

\section{Introduction}

In the context of computing environment for human learning or more specifically in serious collaborative games; Trainers are not face to face with these learners to determine their interactions and questions; if they are well assimilated the knowledge presented. This is why it is necessary to adapt and improve training by gathering as much information as possible about learners. To achieve this goal, much research on the analysis and interpretation of the activities carried out by the learners during their gaming sessions, has been conducted. This work deals with the collection and analysis during games, information called "traces". The exploitation of these traces, defined as temporal sequence of observation, setting up activity knowledge to be able to adapt it to the learner level. However, the majority of applications personalize doesn't even take into account the learning style of each learner. Previous studies have indicated that without basic models that take learners' needs or learning difficulties into account, learners may only show a temporary interest in the learning process and their learning outcomes are not assured.

Many studies have shown that, , learning systems can be more beneficial to learners by taking into account learning styles, due to the personalized presentation of learning content that corresponds to the perceived information and the treatment styles of individuals (Soflano 
et al., 2015). Most of learning styles have been proposed with the intention of understanding the differences between heterogeneous learners and to enable learners to be classified as a specific group. Researchers have proposed several learning styles such as Kolb, Honey, Felder \& Silverman and many more. Majority of approaches that were carried out in this field are based on three parts: the first is to identify the learning style for each learners by doing a survey and a pre-test to determinate their knowledge level. The second consist to implement an adaptive system either dynamic according to learners interactions or presentation of personalized game version according to information collected from survey, and the last part presented in the form of a survey (post-test) to measure effectiveness in order to approve the model. Adaptation had the potential to improve the effectiveness of learning in a game-based learning GBL (Tseng et al. 2008). In addition, Felder-Silverman learning model is the most used model; it represents elements from most models, which presents the generality of the model. Moreover, it has been proven to be effective in many adaptive learning systems (Filippidis \& Tsoukalas, 2009; Soflano et al., 2015; Hwang et al., 2012). However, the proposed adaptation models using implicit method and which are not based on formal computerized one (Soflano et al., 2015; Hwang et al., 2012). More than this models adapt the game for a single learner and the majority designed to be controlled by a game master (Wendel et al., 2013).

This paper attempts to develop an adaptive system to personalize a collaborative serious game to the skill level of the learner according their learning style, the proposed model based on the transformation of traces in order to be adjusted to the profile of their target learners and adapt game by using Npc (Non character player). In particular, it covers three issues:

- How to adjust the traces? It consists of transforming the trace of a source learner in order to be adjusted to the target users. For this, a model of representation for traces has been developed; it's based on a model proposed by (Sehaba, 2012) and transformation process (adjustment of traces taking into account the profile of the target learners).

- How to extract the relevant adaptation knowledge from traces? This is to propose a group model in order to present generalization about traces and learners' profiles especially learning styles, to fulfill this requirement some methods are employed.

- How to use Npc to adapt game? This step is released by using agent technology.

The rest of the paper is organized as follows. Section 2 presents a short review of learning style theories followed by a review concerning empirical work in adaptive game based on learning styles, adaptation model based trace and collaborative learning. Section 3 introduces the overall system architecture and describes the proposed method which includes specific models used for trace and the representation of group model, and also the adjustment of traces and adaptation process. Section 4 presents the scenario of the play game. section 5 describes the experimental tests to prove the validity of the system. Finally, section 6 provides the concluding remarks.

\section{Related Work and Literature Survey}

In the past decade, researchers have proposed several learning style models, each proposing different descriptions and classifications of learning types. Learning styles have been considered as an important human factor that affects learning performance (Filippidis and Tsoukalas, 2009). Personalized learning content has been recognized as being one of the most crucial features of educational systems (Tseng et al., 2008).

Among those learning style theories, the Felder-Silverman learning style has been widely used in game based learning applications and has been validated by various studies(Filippidis and Tsoukalas 2009; Hwang et al. 2012; Soflano et al. 2015). Felder-Silverman Learning Styles Model was developed by Richard Felder and Linda Silverman (1988). The model maps the learners into four dimensions: Active and Reflective, Sensitive and Intuitive, Visual and Verbal, and Sequential and Global. The extremes of these dimensions are defined as follow (Franzoni and Assar, 2009):

-Active learner processed the information through physical activities and discussions and likes group work; the reflective through introspection and prefer working alone.

- Sensitive learner tend to receive information in kind of external agent like places sounds and physical sensation; the intuitive through internal agents like possibilities, ideas and enjoy discovering connections between problems.

- Visual learner remembers best anything that have been seen, such images, diagrams, graphics; the verbal gets out of words, both written and spoken.

- Sequential learner adopts problems in stepwise, the global through leaps and an integral approach.

Game based learning or serious game has been recognized as a potential platform in supporting student learning. Many studies have reported that players are motivated and focused while engaged in gaming sessions (Hoffman and Nadelson 2010; Huang et al. 2010). Educators observed that the diversity of research on positive affects and results associated with playing educational games was noteworthy (Hwang et al., 2012).

Furthermore, the personalization process is suggested and it's the most important features of game based learning (Tseng et al., 2008). In such educational game, it is crucial to select appropriate game mechanics, pedagogical 
methods and modalities to support the player preferences, knowledge levels and learning styles (Huang et al. 2010). Adaptive GBL has been defined as "the ability of the GBL application to automatically customize certain elements of the system based on a series of the student's interactions with the system" (Soflano et al., 2015). Two adaptation process can be used in developing such as systems, that is "online adaptation" consists in automatically adapting game in real-time by analyzing the historical interactions and presenting personalized content for individual learners, and "offline adaptation" is applied by providing options that guide learner to personalize the game according to their own preferences (Brusilovsky, 2001).

Several studies have been conducted to develop adaptive game based learning, based on learning styles. For example: (Tseng et al., 2008) developed an adaptive learning game by basing on learning behavior and learning styles. (Carver et al, 1999) proposed adaptive hypermedia interface that provide dynamic tailoring based on individual student's learning style. Recently, (Bourekkache et al., 2017) developed an adaptive system for personalized learning based learning styles and multi agent system. Various works have been based on the Felder-Silverman model, for example, (Filippidis and Tsoukalas, 2009) also developed an adaptive educational system based on learning styles. The adaptive learning system provides fragments of different images that present the same content and it choose the one most relevant to the user based on the sequential/global dimension. (Hwang et al., 2012) developed personalized game-based learning approach based on sequential/global dimension for an elementary school natural science course. Lately, (Soflano et al., 2015) developed an adaptive GBL application to teach basics of the database programming language SQL (Structured Query Language), this application based on visual/verbal dimension.

Capturing and utilizing of learning experiences and traces of learners as knowledge is definitely an important topic and has already leaded to researches in many educational applications (Bousbia et al., 2010; Sehaba, 2012; Karami et al., 2016). For example, Bousbia (2010) proposed a web- based learning environment "Indicator for the Deduction of Styles", it is based on extraction of indicators from traces to indentify learners' behaviors and learning styles automatically. Sehaba (2012) also developed an approach for sharing of experiences in the context of e-learning applications; it is based on models and tools for transforming traces in order to personalize them for the specific needs of their target users. Recently, (Karami et al., 2016) developed an intelligent system (companion robot) to adapt behaviors/actions according user's feedback. It consists in analyzing the traces of interaction between the robot and the users.

Collaborative serious games can be viewed as a powerful way and as the most suitable domain to use technology to support collaborative learning. Various researches
(Boufera and Bendella, 2016)(Afifi et al., 2016) have indicated that collaborative serious game enhances social competencies such as collaborative decision-making, negotiation and communication. Collaboration is defined by (Roschelle and Teasley, 1995) as "a coordinated, synchronous activity that is the result of a continued attempt to construct and maintain a shared conception of a problem". Dillenbourg (1999) defines collaboration as follows: "In collaboration, partners do the work together" (Dillenbourg, 1999). Johnson and Johnson (1994) state the following about collaboration: "Collaboration is a multidimensional, variable construct composed of five key dimensions, two of which are structural in nature (governance and administration), two of which are social capital dimensions (mutuality and norms), and one of which involves agency (organizational autonomy)" (Johnson and Johnson, 1994).

The various definitions of collaboration are used as a basis for a formalization of the concept of collaborative learning, which is widely used in game-based learning GBL. There are several parameters defining the success of collaborative learning. One Most important factor is the group of learners. The group is characterized by its size, and by its composition. Level of competences and preferences should be taken into account when forming groups.

Due to the lack of concepts for adaptation of collaborative serious games, only a limited number of collaborative serious games have been designed with adaptation support. (Victor et al., 2013) proposed a collaborative multiplayer Serious Game prototype. Escape From Wilson Island (EFWI) was developed with an automatic adaptation mechanism and a game master interface (GM), adaptation is based on GM.

\section{System Architecture and Design}

Figure 1 shows a schematic overview of the framework. The approach presented here consists in introducing models and methods to adapt the game to the target users with different profiles P2, P3 by transforming traces shared by a user with profile P1. The collaboration is released in non real time.

The main purpose of the proposed system is to recommend adequate and useful materials to learners based on their learning styles. The system consists of four functional modules: 'trace base', 'group model', 'adaptation knowledge' and 'adaptation engine'.

The trace database presents the storage for all traces shared by learners during game sessions. It describes how the information content is structured.

The Group Model: Is a collection of data about learning styles and properties of learner profiles. Each learner profiles contain personal information, capacities and preferences of learners. The system uses this data to 


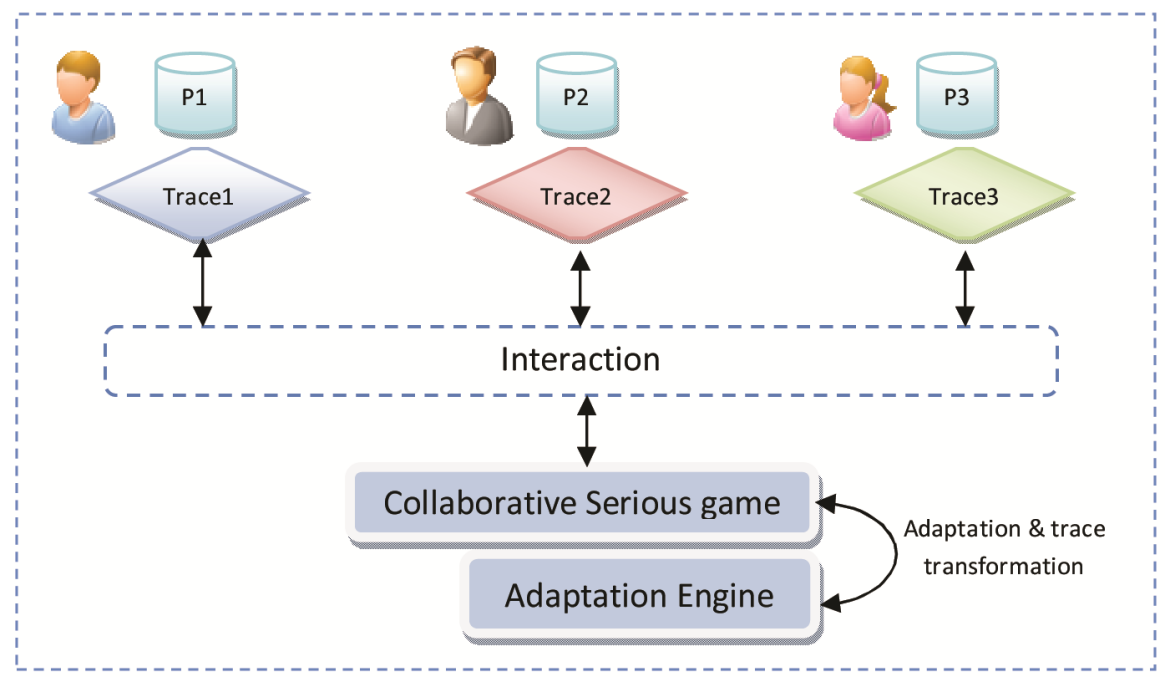

Figure 1. General context of the proposed system

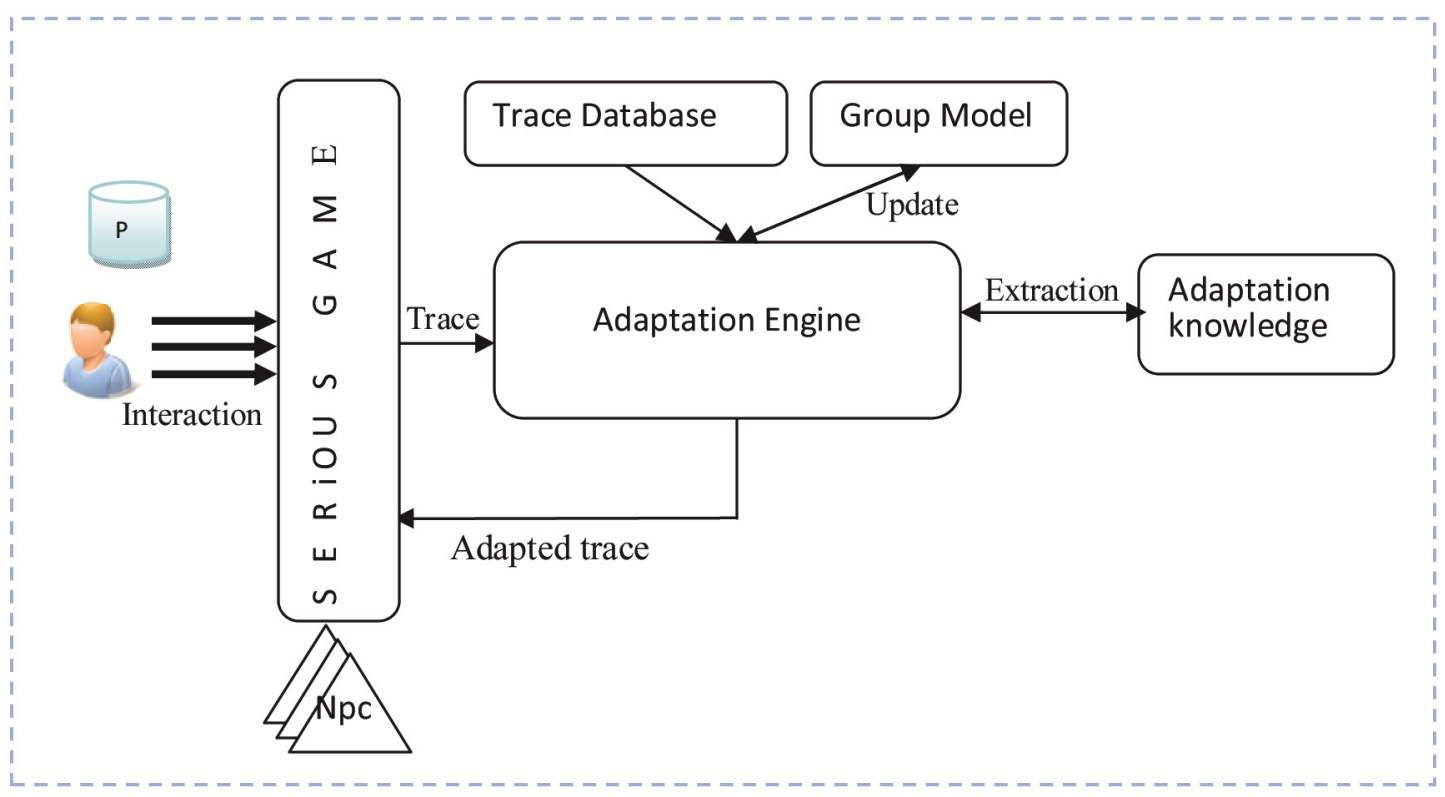

Figure 2. System architecture

extract the adaptation knowledge.

Adaptation Knowledge: Permits to the system to select, for each action of the trace, the adequate mode based on the properties of the learner profile.

Adaptation Engine: Is responsible to update the group model, extraction of adaptation knowledge and it adapt the content and learning materials of the game by using Npc.

The new learner sign up by using the registration form in order to create a personal profile. Once the learner starts a new game session by performing given tasks, the system records its actions and game resources, learning materials to accomplish each task. It represents them in form of trace presented in section3. All traces collected by the system are used to create and update the group model, by selecting for each action the possible mode to achieve it (see section3.3). When the system detect difficulties to perform an action / by a learner, the adaptation engine compare the properties of each mode to the learner profile and extract the adequate learning materials list to perform this action. Finally, Npc adapt the content of the game with executing the best plan constructed from the list of adaptation knowledge.

\subsection{Trace}

A trace $T$ is a sequence of learner actions, and Gaming and Learning Material Indicator (GLMI) used to perform these actions.

$T: T=<l, \operatorname{task},\left(G L M I_{1}, G L M I_{2}, \ldots G L M I_{n}\right)>$.

$\bullet l$ : The traced learner.

- task: The description of learner task. 
- $G L M I_{i}$ : Gaming and Learning Material Indicator, GLMI is a tuple $<A_{i}$, $h \_d i s s, h$ exp, ver_inter, vis_inter, eval_time, Ord>

GLMI presents some indicators to predict learning style dimension like active or reflective extracted from (Franzoni and Assar 2009; Becker 2005), for example: an active learner prefers discussions and group working; he/she helps each other and in the same time need helping from other.

$A_{i}$ is learner action.

$h$ diss learners who help themselves and others by using discussion tool, with $h \_$diss $\in\{0,1\}$.

$h \_\exp$ learners who help themselves by exploring examples, with $h \_\exp \in\{0,1\}$.

ver_inter learners who prefer or not the verbal interaction, with ver_inter $\in\{0,1\}$.

vis_inter learners who prefer or not the visual interaction, with vis_inter $\in\{0,1\}$.

eval_time presents the evaluation of time to performe an action, with eval_time $\in[0,1]$. For example eval_time $=1$ if the time for perform an action is suitable.

Ord presents the order of the performed action, with $\mathrm{Ord}=$ $\{0,1\}$. For example Ord $=1$ if the action 1 in scenario has performed firstly else $\mathrm{Ord}=0$.

\subsection{Learner Profile}

Learner profile $(L P)$ describes capacities, responsibilities and preferences of learner. It gives important information to adapt the learning style to the target learner. In this work, especially social and motivation capacities, verbal and visual preferences have exploited.

$L P$ is a set of properties that characterize the learner:

$$
L P=\left\{p_{1}=v_{1}, p_{2}=v_{2} \ldots p_{n}=v_{n}\right\}
$$

$p_{i}$ : The property description, properties represent capacities (social, motivation) and preferences (verbal, visual).

$v_{i}$ : A value of each property, with $v_{i} \in[0,1]$. For example, the social capacity of a learner who prefers working in group is social_cap $=1$, and for learner prefers work alone is social_cap $=0$.

\subsection{Group model}

Group model represents the state of all learners, it describes classes of actions effected by learners, each class contains sub classes represents all GLMI indicators, each indicator is defined by a Mode. The Mode defines the conditions under which the GLMI indicator can be used. $S$ presents a threshold defined by an expert. It used to select the useful properties and add it to the mode.

Algorithm1. Algorithm to update a group model.
For all action $A_{i}$ do

$$
\begin{aligned}
& A_{i} \leftarrow\left\{G L M I_{1}, G L M I_{2} \ldots G L M I_{n}\right\} \\
& \text { For all GLMI do } \\
& G L M I_{i} \leftarrow \text { Mode }_{i} \\
& \text { For } i=1 \text { to } n b_{-} \text {GLMI do } \\
& \text { For } j=1 \text { to } n b_{-} p d o \\
& \text { If } v j>=S_{j} \text { then } \\
& \text { Mode }_{i} \leftarrow p_{j} \\
& \text { End }_{i f} \\
& \text { End }_{\text {for }} \\
& \text { End }_{\text {for }} \\
& \text { End }_{\text {for }} \\
& \text { End }_{\text {for }}
\end{aligned}
$$

\subsection{Adaptation Process \& Knowledge Adaptation}

Knowledge adaptation permits to the adaptation process to select, for each action the adequate GLMI indicators based on properties of learner profile, to adapt their learning style.

Extraction of knowledge adaptation represents the important step in the proposed approach model. The objective is to present the target learner a personalization of different gaming and learning materials by finding the properly GLMI indicators:

- Search in learner model, exactly in actions classes $A_{i}$ all $G L M I_{i}$ indicators sub-classes with all mode .

- Compare the properties of the profile of target learners with the properties $p_{i}$ of all mode ${ }_{i}$ and determine those with similar values.

- Determine $G L M I_{i}$ indicators with the selected mode ${ }_{i}$.

- Assign the actions $A_{i}$ with the right $G L M I_{i}$ indicators to the plan, in order to be executed by an $N P C$ player.

\section{Adaptive Collaborative Serious Game Based Learning Style}

In this research, a game for simulation a comportment of fire commander is developed and selected to test the proposed approach. Figure 3 shows the storyline that requires the room to be accessed, the fire to be extinguished and the victim to be saved. The blocks in the figure 3 represent subscenes. The aim of the game is to train the learner to accomplish these tasks:

- Get access to a room (T1)

- Extinguish fire (T2)

- Evacuate victim (T3)

In this game (figure 4), learners simulate firemen with their 


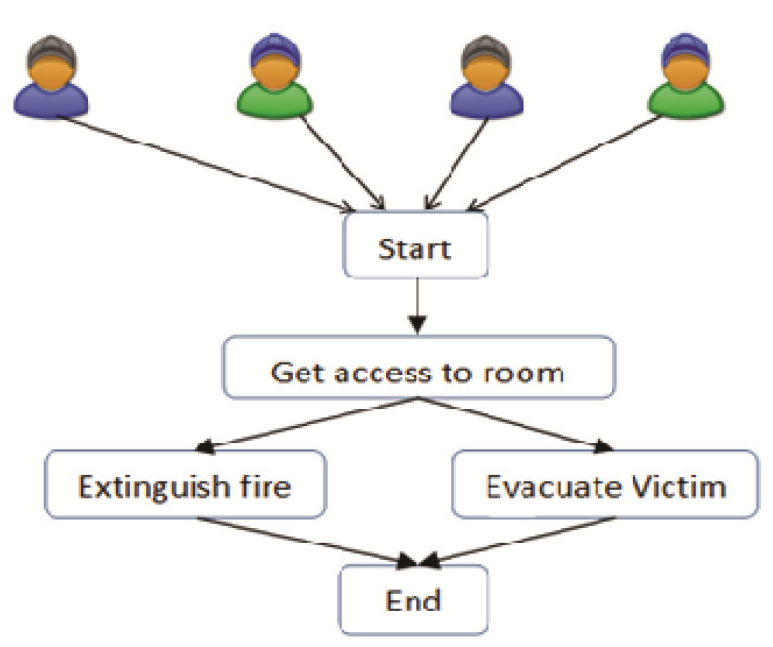

Figure 3. Scenario of the playgame

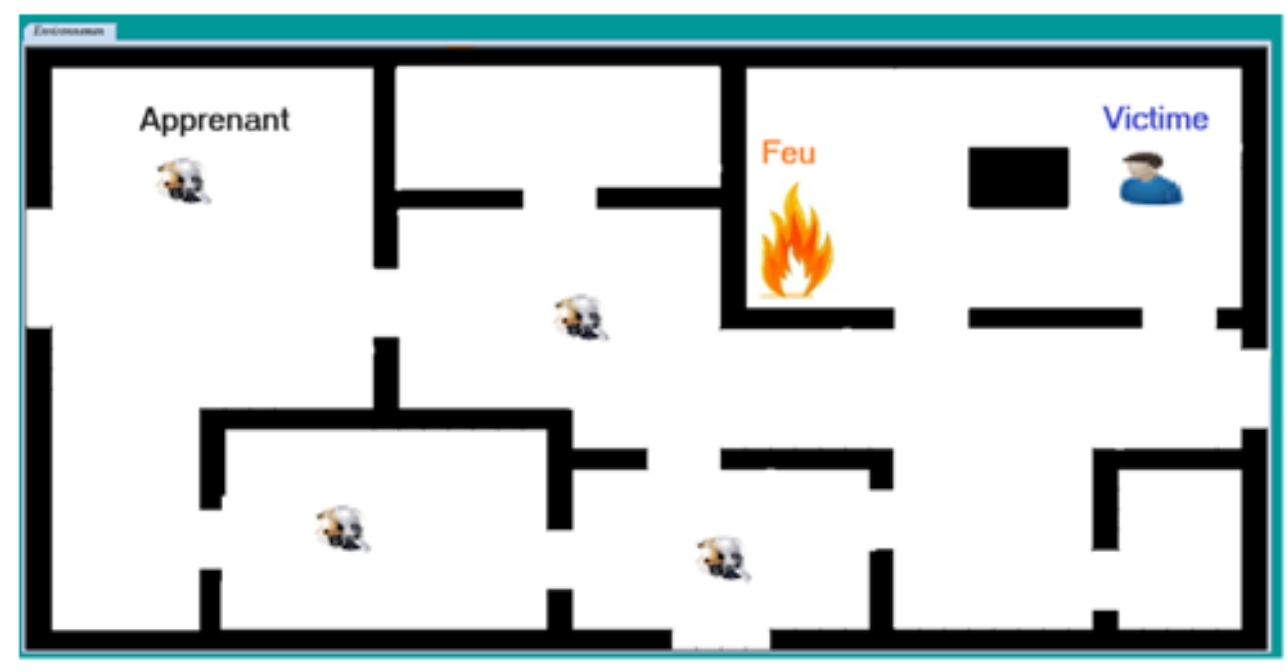

Figure 4. Screenshot of the developed game

own preferences, and skills. The evaluation on performance is done when the learner complete each task. Otherwise, when learner has difficulties to do the task, adaptation process is triggered automatically to help and adapt the target learner to achieve it taking into account their learning style.

In this study, there were two mode of the same game developed and tested:

- An adaptive mode based on learning style (ABLSG): The game has an adaptive system to customize the game to the learners based on their learning style.

- An adaptive game without taking into consideration the learning style $(A B G)$ : This mode personalizes the collaborative serious game to the skill level of learners, and it doesn't takes into account learner's learning styles.

\section{Experiment Method and Results}

The main purpose of this research is to evaluate whether the use of the game with adaptivety based on learner's learning style can improve learning comparing to the use of one without taking into consideration the learning style. Therefore, two groups are suggested in this experience:

- Experimental group with learners guided by the first mode of the game (ABLSG).

- Control group that will play the second mode of the game (ABG).

The experiment included undergraduate students from the University of Mascara Algeria. All of participants are from technical science program. A total of thirty two students participated in the study, a group of seventeen students represent the experimental group and the others constitute the control group.

All of students were not formed on game story before running the game. During the game, the interactions between the learners and the game were recorded automatically to identify the learner's learning style during 
the game. A game score is used to evaluate learner performance.

\subsection{Analysis of Learning Outcomes (Game Score)}

The aim of this study was to examine the effectiveness of the proposed system in improving the learning outcomes of learners. The mean values and standard deviations of game evaluation scores are 25.76 and 5.77 for the experimental group, and 18.26 and 7.06 for the control group. The $t$ test result $(t=3.26, p>0.05)$, there is a significant difference between the two groups; that is, the learners who played with the ABLSG version showed significant better learning outcomes than those who learned with the $A B G$ version.

\begin{tabular}{|l|c|l|l|l|}
\hline & $N$ & Mean & $S D$ & $t$ \\
\hline Experimental group & 17 & 25.76 & 5.77 & 3.26 \\
\hline Control group & 15 & 18.26 & 7.06 & \\
\hline
\end{tabular}

Table 1. $t$ test result of game score

\subsection{Completion Times Analysis}

This analysis includes the comparison between experimental group and control group. The completion times is the time required for the learner to complete the game. This completion times is recorded automatically during the game session. The completion times when learning through the ABLSG mode was $23.29 \mathrm{~min}$ ( $\mathrm{SD}=$ 9.20 ), while the mean completion times for $A B G$ mode was $38.06 \mathrm{~min}(\mathrm{SD}=10.8)$. The $t$ test $\mathrm{t}(t=4.11, p>0.05)$ showed the completion times for the experimental group was shorter than the control group.

\begin{tabular}{|l|c|c|l|l|}
\hline & $N$ & Mean & $S D$ & $t$ \\
\hline Experimental group & 17 & 23.29 & 9.20 & 4.11 \\
\hline Control group & 15 & 38.06 & 10.8 & \\
\hline
\end{tabular}

Table 2. $t$ test result of completion times

\section{Correlation Between Completion Times and Learning Outcomes}

To study the correlation between completion times and learning outcomes, Pearson-correlation test is used. The analysis shows that the correlation between completion times and learning outcomes in the control group $(r=-$ $0.28)$ is weak. However, the experimental group $(r=0.19)$ has a higher correlation between completion times and learning outcomes.

\subsection{Efficiency Analysis of the Adaptive System}

To get a subjective evaluation of the proposed system, the system affect a predict rating $\left(p_{i}\right)$ at each plan executed by the Npc player, thereafter a real rating $\left(q_{i}\right)$ is associated to the reaction of the learner, for example: If a learner adopt and follow the plan of Npc player, the difference between the real and predictive rating is negligible.

A mean absolute error (MAE) is used to assessing accuracy of the proposed system. It is calculated at each task (T1, T2, and T3) for the experimental group.

$$
M A E=\left(\sum_{i=1}^{n}\left|p_{i}-q_{i}\right|\right) / N
$$

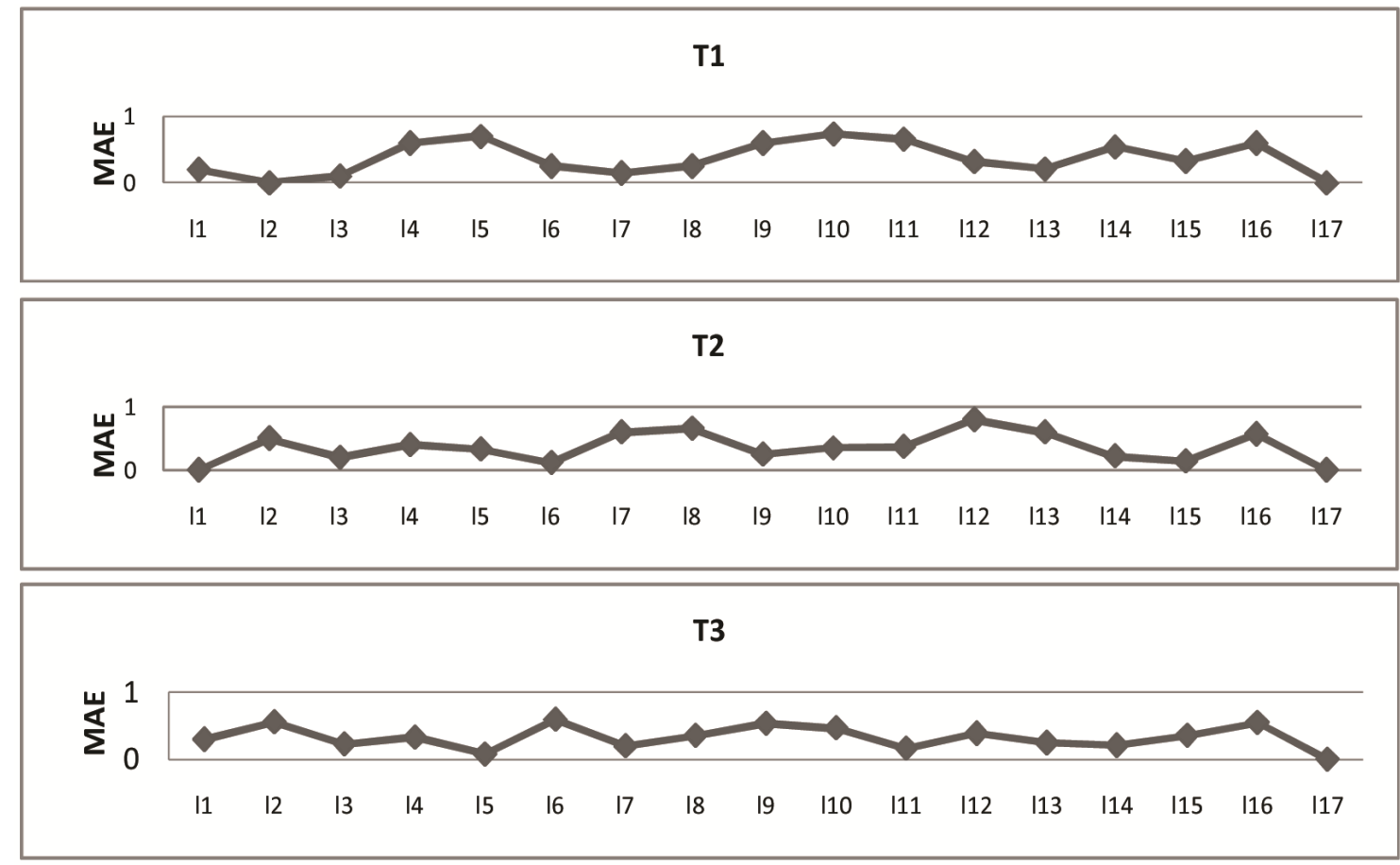

Figure 5. The result MAE for the 3 tasks 
The lower the MAE, the More the accurately adaptation system predicts learner plans and reactions. From the figure 5, the MAE is well under 1.0, which means that the proposed system is accurate and it makes learning more efficient.

\subsection{Motivation Analysis}

When learners start session of game, they indicate if they are motivate to play the game and in the end of session they are asked if they are still motivated or not. the results show that before playing the game, the two groups have equivalent learning motivation.

After they finished, it is found that the learning motivation of experimental group is largely higher than that of the control group.

\begin{tabular}{|l|c|c|}
\hline & $\begin{array}{c}\text { Motivation after } \\
\text { game (\%) }\end{array}$ & $\begin{array}{c}\text { Motivation before } \\
\text { game (\%) }\end{array}$ \\
\hline Experimental group & 90 & 92 \\
\hline Control group & 90 & 54 \\
\hline
\end{tabular}

Table 2. Learning motivation rate

\section{Discussion and Conclusion}

Adaptive collaborative serious game needs to consider learner's specific competences and requirements like her/ him learning style.

This paper presents an approach for adapting collaborative serious game based on learning style; the developed system can personalize the game to learner by transforming traces of other learners and extracting knowledge adaptation in order to form a plan that will be executed by Npc player. Several experiments have been carried out to evaluate the system.

The results shows that the adaptive collaborative serious game that takes into account the learner's learning style significantly benefited the learner.

In term of learning outcomes, the study found that the ABLSG version enables participants of experimental group in a way that customize the game in accordance with their learning style, their learning outcomes are higher compared to those of learners in control group. This finding is pursuant to previous studies (Hwang et al. 2012; Soflano et al. 2015). To sum up, taking into consideration the learning styles and the adaptation process in collaborative serious game has boost the effect of the game proposed.

Moreover, when comparing the completion times of each group, there is a large difference between the two groups. The control group takes more time to complete a game than the other group. beside, the results shows a positive correlation between completion times and learning outcomes. These finding confirm the importance of adapting the game. The ABLSG has the advantage that personalizes the game elements by filtering learning materials, it helps to shorten the learner's completion times.

Furthermore, from the aspect of motivation, this study shows that the learning motivation can be pushed when the learning style is taking into consideration. Moreover, the proposed system has shown important efficiency and accuracy in improving learning for different learners.

From results obtained, it turns to be more advantages from the findings of researches conducted by Soflano et al. (2015) and Hwang et al. (2012), it showed by:

- The proposed adaptive system is detailed and well structured; it comprises modules and methods to select and extract information about learners, and tailors the game to their specific learning style.

- The proposed adaptivity is improved, it address multiple learning styles dimensions, contrary to the research proposed by Soflano et al. (2015) and Hwang et al. (2012) that treat just one dimension.

In the future, we plan to evaluate the system of this study with large-scale experiments; moreover, we shall develop the proposed approach by providing more indicators.

\section{References}

[1] Soflano, M., Connolly, T. M., Hainey, T. (2015). An application of adaptive games-based learning based on learning style to teach SQL, Computers \& Education, 86, p. 192-211.

[2] Bousbia, N., Labat, J.-M., Balla, A., et Rebaï, I. (2011). Supervised classification on navigational behaviours in web-based learning systems to identify learning styles. International Journal of Learning Technology, Inderscience (IJLT), 6 (1) 24-45

[3] Tavassolian, A., Stanley, K. G., Gutwin, C. (2014). The effect of temporal adaptation granularity and game genre on the time-balancing abilities of adaptive time-varying Minigames. Entertainment Computing, 5, 43-54.

[4] Hwang, G.-J., Sung, H.-Y., Hung, C.-M., Huang, I. (2013). A Learning Style Perspective to Investigate the Necessity of Developing Adaptive Learning Systems. Educational Technology \& Society, 16 (2)188-197.

[5] Brusilovsky, P. (2001). Adaptive hypermedia. User Modeling and User Adapted Interaction, 11, 87-110.

[6] Tseng, Judy, C. R., Chu, H. C., Hwang, G. J., Tsai, C. C. (2008). Development of an adaptive learning system with two sources of personalization information. Computers \& Education, 51 (2) 776-786.

[7] Carver, C. A., Howard, R. A., Lane, W. D. (1999). 
courseware and incorporation of student learning styles. IEEE Transactions on Education, 42 (1) 33-38.

[8] Bourekkache, S., Kazar, O., Kahloul, L., Tigane, S., Benharkat A-N. (2017). Educative and Adaptive System for Personalized Learning: Learning Styles and Content Adaptation. International Arab Journal of Information Technology (IAJIT), 4 (3) 125-132.

[9] Filippidis, S. K., Tsoukalas, L. A. (2009). On the use of adaptive instructional images based on the sequentialglobal dimension of the Felder-Silverman learning style theory, Interactive Learning Environments, 17 (2) 135150.

[10] Wendel, V., Gutjahr, M., Göbel, S., Steinmetz, R (2013).Designing Collaborative Multiplayer Serious Games, Education and Information Technologies, 18 (2), p. 287-308.

[11] Karami, A-B.., Sehaba, K., Encelle, B. (2016). Adaptive Artificial Companions learning from users' feedback, Adaptive Behavior Journal, p.1-22.

[12] Sehaba, K. (2012). Système d'aide adaptatif à base de traces. Revue internationale des technologies en pédagogie universitaire. 9 (3).

[13] Franzoni, A. L., Assar, S. (2009). Student Learning Styles Adaptation Method Based on Teaching Strategies and Electronic Media. Educational Technology \& Society, 12 (4)15-29.

[14] Huang, W. H., Huang, W. Y., Tschopp, J. (2010). Sustaining iterative game playing processes in DGBL:
The relationship between motivational processing and outcome processing. Computers \& Education, 55 (2) p.789-797.

[15] Hoffman, B., Nadelson, L. (2010). Motivational engagement and video gaming: A mixed methods study. Educational Technology Research and Development, 58 (3) 245-270.

[16] Boufera, H., Bendella, F. (2016). Simulation for Collaborative Serious Game Using Player's Modeling and Agent Technology, Int'l Journal of Computing, Communications \& Instrumentation Engg. (IJCCIE), 3 (2).

[17] Dillenbourg P. (1999). What do yuo mean by collaborative leraning?. In: P. Dillenbourg (Ed) Collaborative-learning: Cognitive and Computational Approaches. p.1-19.

[18] Johnson, D. W., Johnson, R. T. (1994). Learning together. In: Sharan S, editor. Handbook of cooperative learning methods.

[19] Roschelle, J., Teasley, S. D. (1995). The construction of shared knowledge in collaborative problem solving, In: C.E. O'Malley (Ed), Computer-Supported Collaborative Learning. p. 69-197.

[20] Becker, K. (2005). Games and Learning Styles, Proc. ICET2005, Calgary, Alberta, Canada Lafifi, Y., Mehira, N., Zedadra, A.(2016). Dynamic grouping of learners in a computer- supported collaborative practicalworks system, International Journal of Knowledge-based and Intelligent Engineering Systems, 20 (1), 37-48. 